\title{
The relationship between mean platelet volume and platelet levels of children with Helicobacter pylori and gastritis
}

\author{
Mehmet Agin ${ }^{1}$, Yusuf Kayar ${ }^{2}$, Ramazan Dertli² \\ ${ }^{1}$ Department of Paediatric Gastroenterology, Van Education and Research Hospital, Van, Turkey \\ 2Department of Gastroenterology, Van Education and Research Hospital, Van, Turkey
}

Key words: mean platelet volume, platelet, Helicobacter pylori, gastritis.

Address for correspondence: Mehmet Agin, Department of Paediatric Gastroenterology, Van Education and Research Hospital, Van, Turkey, phone: +90 5068011083, e-mail: drmehmet47@yahoo.com

\begin{abstract}
Introduction: Helicobacter pylori (HP) is an infectious agent worldwide and is detected frequently.

Aim: We examined whether there is a relationship between HP positivity and gastritis, mean platelet volume (MPV), and platelet level.

Material and methods: A total of 309 patients who presented to the Paediatric Gastroenterology Polyclinic with dyspeptic complaints, who received upper gastrointestinal endoscopy, and whose gastric biopsies were carried out were included in the present study. MPVs, thrombocyte, presence of HP, and histopathological presence of gastritis in the patients were examined. The MPV and thrombocyte values were compared between the patients who were HP positive and negative, and between the cases who had and who did not have gastritis.

Results: A total of $36 \%$ of the cases who were included in the present study were $\mathrm{HP}(-)$, and $64 \%$ were $\mathrm{HP}(+)$. No gastritis was detected in $22 \%$ of the cases. No significant differences were detected between the HP(+) and HP(-) cases in terms of MPV values. The platelet values were determined to be low at a significant level in the $\mathrm{HP}(+)$ group, although no significant differences were detected between all the cases who had and who did not have gastritis in terms of MPV.

Conclusions: A weak relation was determined between the HP and MPV and platelet values. Although no relations were detected between the presence of gastritis or its severity and MPV, a weak relation was detected between the presence of gastritis and platelet values.
\end{abstract}

\section{Introduction}

Helicobacter pylori (HP) is a common infectious factor all over the world. It is known that this infection increases with low socioeconomic level and family structure. HP infection is seen more frequently and earlier in developing countries compared to developed countries. Mostly, HP is associated with gastrointestinal infection, and is one of the major aetiological causes of gastric malignancies, mucosa-related lymphoma, peptic ulcer, and chronic gastritis [1].

Thrombocytes are specialised blood cells playing primary roles in haemostasis, thrombosis, and coagulation. This may be examined during automatic whole blood count without incurring any extra costs. The mean platelet volume (MPV) is an indicator of the platelet function and activation and is measured with clinical haematology analysis [2]. Thrombocyte volume measurements are objective measurements in evaluating the thrombocyte size. Increasing MPV is associated with an increase in the megakaryocytic growth in response to the thrombopoietic stress response [3]. If there is inflammation in the body, some cytokines and chemokines are released from the thrombocyte membranes $[4,5]$. In this way, during the inflammation, platelet activation may be measured indirectly with MPV. The measurement of MPV is easy and cheap. It was shown in previous studies that MPV is a useful method in evaluating thrombocyte function and activation [6]. Inflammation is an important stimulus for thrombocytes. MPV was examined in many diseases as a simple inflammatory marker. Thrombocyte volume and structural chang- 
es might help in the differential and early diagnosis of several diseases. Several studies examined the relation between MPV and some diseases [7-13].

\section{Aim}

In this study, it was examined whether there is a relation between the presence of HP and gastritis, MPV, and platelet count.

\section{Material and methods}

A total of 309 patients who applied to the Healthcare Sciences University, Van Education Hospital, Paediatric Gastroenterology Polyclinic between October 2017 and October 2018, who had dyspeptic complaints and who received upper gastrointestinal system endoscopy and gastric biopsy were included in the present cross-sectional study. Those who had systemic diseases or received treatment for systemic diseases, those who used drugs that affected thrombocyte functions, those who had another inflammatory or infectious disease, thyroid disease, malignity, renal, cardiac, and liver failures, diabetes mellitus, hypertension, inflammatory bowel disease, and those who had haematological diseases were excluded from the study. Biochemical measurements and biochemical parameters were measured from the antecubital venous blood samples that were taken in the morning hours after eight hours of fasting. MPV measurements were made within 30 min after the blood samples were taken. The samples were collected with tubes that included dipotassium EDTA. In the present study, the complete blood count analysis was made with an automatic haematology analyser: Beckman Coulter LH 750 (Beckman Coulter, USA). Thrombocyte and MPV levels were also measured. Age, gender, MPV, thrombocytes, the presence and intensity of HP, and the presence of histopathological gastritis and its severity were investigated. Gastritis was classified according to the Modified Sydney System [14]. The presence of HP was recorded as $1(+), 2(+)$, or $3(+)$, and the presence of gastritis was interpreted as no gastritis, mild, moderate, and severe gastritis. The MPV and thrombocyte values were compared in patients who were HP positive and HP negative and who did not have gastritis.

\section{Statistical analysis}

Normal distribution was tested by using the Shapiro-Wilk test. Mann-Whitney $U$ test was used to compare non-normal variables between two independent groups. Spearman's rank correlation coefficient was used to test the relationships between numerical variables. ROC curve analysis was performed to determine the diagnostic accuracy of numeric variables. Mean \pm standard deviation for numeric variables and frequency and percentages for categorical variables were given as descriptive statistics. SPSS 24.0 software package was used in the analyses. $P<0.05$ was accepted as being statistically significant.

\section{Results}

A total of $35 \%(n=109)$ of the patients were male and $65 \%(n=200)$ were female. The average age of the cases included in the present study was $12 \pm 6$ years. A total of $36 \%$ of the cases were $\mathrm{HP}(-)$ and $64 \%$ were $\mathrm{HP}(+)$. A total of $29 \%$ of the HP positive cases were $1(+), 24 \%$ were $2(+)$, and $11 \%$ were $3(+)$. No gastritis was detected in $22 \%$ of the cases. Mild gastritis was detected in $38 \%$, moderate gastritis was detected in $30 \%$, and severe gastritis was detected in $10 \%$ of the cases (Table I). No significant differences were detected in terms of MPV values between $\mathrm{HP}(+)$ and $\mathrm{HP}(-)$. The platelet values were detected to be low in the $\mathrm{HP}(+)$ group at a significant level $(p=0.038)$. ROC curve analysis was carried out to determine the diagnostic value of the platelets in differentiation between the $\mathrm{HP}(+)$ and $\mathrm{HP}(-)$ groups. The AUC value was measured to be 0.572 . This value shows that platelets have a weak distinguishing characteristic. In the ROC analysis, the specificity was determined to be $65 \%$ (95\% CL: $55.4-74)$, and the sensitivity was determined to be $49.5 \%$ (95\% CL: 42.4-56.6). The MPV measurements were made between $\mathrm{HP}(+)$ and $\mathrm{HP}(-)$ subgroups, and a statistically significant difference was detected between the $\mathrm{HP}(2+)$ and $\mathrm{HP}(-)$ groups $(p=0.022)$. The MPV values were high in the $\mathrm{HP}(2+)$ group at a significant level. Again, the same relation was observed for platelet level

Table I. Helicobacter pylori, gastritis, and gender frequency in the cases receiving endoscopy

\begin{tabular}{lll} 
Variables & $\boldsymbol{N}$ & $\%$ \\
\hline H. pylori: & 110 & 36 \\
\hline Negative & 91 & 29 \\
\hline $1(+)$ & 74 & 24 \\
\hline $2(+)$ & 34 & 11 \\
\hline $3(+)$ & 109 & 35 \\
\hline Gender: & 200 & 65 \\
\hline Male & & 22 \\
\hline Female & 67 & 38 \\
\hline Gastritis: & 117 & 30 \\
\hline Negative & 93 & 10 \\
\hline Mild & 32 & \\
\hline Moderate & & \\
\hline Severe & 32 & \\
\hline
\end{tabular}


$(p=0.017)$. A statistically significant relation was determined between the platelet and MPV values in all groups. This result means that as the MPV value increases, the platelet value decreases. The ROC curve analysis was performed for the purpose of evaluating the diagnostic value of MPV in distinguishing between the $\mathrm{HP}(2+)$ and $\mathrm{HP}(-)$ groups. The sensitivity was determined to be $87 \%$ (AUC $=0.600 \pm 0.042, p=0.0175$ ), and the specificity was determined to be $31.8 \%$. In the ROC curve analysis that was made for platelet levels, the specificity was determined to be $65 \%$ and the sensitivity was determined to be $56.7 \%$ (Table II).

Although no significant differences were detected in terms of MPV in all patients who had and who did not have gastritis, a significant difference was detected in terms of platelet count between the groups $(p=0.049)$. In the ROC curve analysis that was made to evaluate the diagnostic value of platelet levels in the gastritis (-) and gastritis (+) groups, the specificity was determined to be $74 \%$, the sensitivity was determined to be $42.5 \%$, and a weak relation was detected. In the comparison that was made between the non-gastritis patients and the subgroups that had various severity levels, a difference was observed between the gastritis $(-)$ and moderate-severe gastritis groups only in terms of platelet values ( $p=0.038)$. When the diagnostic distinctiveness of this test was analysed (AUC $=0.596$ $\pm 0.045, p=0.032$ ), it was interpreted as a poorly distinguishing characteristic (Table III).

\section{Discussion}

Platelets play active roles in haemostasis and in the regulation of inflammatory processes [9]. In recent years, numerous studies have been conducted on the clinical significance of MPV. An increase in MPV is accepted as a marker of inflammation; it is also related to platelet activation $[15,16]$. There are controversial results about MPV and inflammatory diseases in the literature. Although the MPV value increases in some inflammatory diseases, it decreases in others, and although MPV increases in tissue oxygenation and malnutrition [11-13, $17,18]$, it was reported that MPV is low in inflammatory bowel diseases, pneumonia, and in the acute stage of familial Mediterranean fever [19-22]. In addition, it was also reported that MPV is increased at a significant level in other inflammatory diseases like rheumatoid arthritis, ankylosing spondylitis, and Behcet's disease and in the active stages of these diseases [17, 23, 24]. In the present study, it was determined that the platelet count was low in patients who had HP positivity. In the ROC curve analysis, we determined a poor relation between the HP positivity and platelet levels, with a sensitivity of $49 \%$ and a specificity of $65 \%$. We did not determine any relations between $H$. pylori positivity and MPV. The patients who had moderate level $\mathrm{HP}(+2)$ positivity had high MPV values. A weak relation was detected in the ROC curve analysis in this respect. As it was shown by Aktas et al. article conducted on gastrointestinal system, MPV was reported as high in patients who had irritable bowel syndrome [25], and MPV was low in patients who had inflammatory bowel diseases, rotavirus gastroenteritis, cystic fibrosis, acute pancreatitis, and acute hepatitis A infection [7-10]. Topal et al. and Yeniova et al. determined that there are no relations between $\mathrm{HP}$, platelet levels, and MPV $[26,27]$. In a study conducted by Umit et al. higher MPV levels in HP-positive patients was reported [28]. Ugras et al. conducted a study and reported that HP did not affect MPV; however, MPV values were lower in patients who had HP-related gastritis compared to the participants in the control group [29]. Although there are very few studies conducted in this field, different results were reported in different studies.

Table II. Comparison of the MPV and platelet values between the HP-negative and HP-positive cases

\begin{tabular}{lccccccccc} 
Parameter & $\mathrm{HP}(+)$ & $\mathrm{HP}(-)$ & $\boldsymbol{P}^{*}$ & $\mathrm{HP}(+)$ & $\boldsymbol{P}^{* *}$ & $\mathrm{HP}(2+)$ & $\boldsymbol{P}^{\star * *}$ & $\mathrm{HP}(3+)$ & $\boldsymbol{P}^{* * * *}$ \\
\hline MPV & $9.31 \pm 1.06$ & $9.09 \pm 1.24$ & 0.281 & $9.15 \pm 1.12$ & 0.647 & $9.53 \pm 0.98$ & 0.022 & $9.2 \pm 0.87$ & 0.882 \\
\hline Platelet & $325251 \pm 263617$ & $332596 \pm 93237$ & 0.038 & $314197 \pm 77522$ & 0.309 & $349121 \pm 42315$ & 0.017 & $303823 \pm 66763$ & 0.193
\end{tabular}

$P^{*}$ - The $p$-value between the HP-positive and HP-negative cases, $P^{* *}$ - the $p$-value between the HP(1+) and HP(-) cases, $P^{* * *}-$ the $p$-value between the $H P(2+)$ and $H P(-)$ cases, $P^{* * * *}$ - the $p$-value between the HP(3+) and $H P(-)$ cases.

Table III. Comparison of MPV and platelet values between the gastritis-negative and gastritis-positive cases

\begin{tabular}{|c|c|c|c|c|c|c|c|c|c|}
\hline Parameter & $\begin{array}{c}\text { Gastritis } \\
\text { negative } \\
(n=67)\end{array}$ & $\begin{array}{l}\text { Gastritis } \\
\text { positive } \\
(n=242)\end{array}$ & $P^{\star}$ & $\begin{array}{c}\text { Gastritis (mild) } \\
(n=117)\end{array}$ & $P^{\star *}$ & $\begin{array}{c}\text { Gastritis } \\
\text { (moderate) } \\
(n=93)\end{array}$ & $P^{\star \star \star}$ & $\begin{array}{l}\text { Gastritis } \\
\text { (severe) } \\
(n=32)\end{array}$ & $P^{\star \star \star *}$ \\
\hline MPV & $9.06 \pm 1.03$ & $9.28 \pm 1.15$ & 0.125 & $9.19 \pm 1.29$ & 0.373 & $9.39 \pm 1.02$ & 0.057 & $9.28 \pm 0.97$ & 0.323 \\
\hline Platelet & $335238 \pm 92246$ & $325794 \pm 242786$ & 0.049 & $317164 \pm 80773$ & 0.033 & $306924 \pm 85566$ & 0.038 & $412187 \pm 634865$ & 0.252 \\
\hline
\end{tabular}

$P^{*}$ - the $p$-value between the gastritis-positive and gastritis-negative cases, $P^{* *}$ - the $p$-value between the gastritis (mild) and gastritis-negative cases,

$P^{* * *}$ - the $p$-value between the gastritis (moderate) and gastritis-negative cases, $P^{\star * * *}$ - the $p$-value between the gastritis (severe) and gastritis-negative cases. 
In the present study of ours, no relations were detected between gastritis severity and the presence of gastritis and MPV. The platelet values were lower in patients who had gastritis. We detected a poor relation in the ROC curve analysis of the patients who did not have gastritis and those who had mild and moderate-severe gastritis, in terms of platelet levels. In a study by Topal et al., they reported lower MPV levels in patients who had moderate and severe gastritis [26]. Ugras et al., on the other hand, detected no relation between gastritis and MPV, which is similar to our study [29].

\section{Conclusions}

As a result, a weak relation was detected between the MPV and platelet values in patients who applied with dyspeptic complaints. Although no relation was detected between the presence or severity of gastritis and MPV, unlike other inflammatory and infectious diseases, which was also reported in previous studies, a poor relation was detected between the presence of gastritis and platelet values.

\section{Conflict of interest}

The authors declare no conflict of interest.

\section{References}

1. Lancaster-Smith MJ, Perrin J, Swarbrick ET, Wright JT. Celiac disease and autoimmunity. Postgrad Med J 1974; 50: 45-8.

2. Both PM, Butterworth RJ. Platelet size: measurement, physiology and vascular disease. Blood Coagul Fibrinolysis 1996; 7: 157-61.

3. Tompson CB, Love DG, et al. Platelet size does not correlate with platelet age. Blood 1983; 62: 487-94.

4. Brandt E, Ludwig A, Petersen F, Flad HD. Platelet derived CXC chemokines: old players in new games. Immunol Rev 2000; 177: 204-16

5. Mayssa M. Zaki. Parasite platelet interactions. PUJ 2011; 4: 127-36.

6. Khandekar MM, Khurana AS, Deshmukh SD, et al. Platelet volume indices in patients with coronary artery disease and acute myocardial infarction: an Indian scenario. J Clin Pathol 2006; 59: 146-9.

7. Uysal P, Tuncel T, Olmez D, et al. The role of mean platelet volume predicting acute exacerbations of cystic fibrosis in children. Ann Thorac Med 2011; 6: 227-30.

8. Almis H, Bucak IH, Celik V. Mean platelet volume in hepatitis A. Eur Rev Med Pharmacol Sci 2016; 20: 2310-4.

9. Beyazit $\mathrm{Y}$, Sayilir A, Torun S, et al. Mean platelet volume as an indicator of disease severity in patients with acute pancreatitis. Clin Res Hepatol Gastroenterol 2012; 36: 162-8.

10. Mete E, Akelma AZ, Cizmeci MN, et al. Decreased mean platelet volume in children with acute rotavirus gastroenteritis. Platelets 2014; 25: 51-4.

11. Zuberi BF, Akhtar N, Afsar S. Comparison of mean platelet volume in patients with diabetes mellitus, impaired fasting glucose and non-diabetic subjects. Singapore Med J 2008; 49: 114-6.

12. Martin JF, Bath PM, Burr ML. Influence of platelet size on outcome after myocardial infarction. Lancet 1991; 338: 1409-11.

13. Ghahremanfard F, Asgharı N, Ghorbanı R, et al. The relationship between mean platelet volume and severity of acute ischemic brain stroke. Neurosciences (Riyadh) 2013; 18: 147-51.

14. Price AB. The Sydney System: histological division. J Gastroenterol Hepatol 1991; 6: 209-22.

15. Endler G, Klimesch A, Sunder Plassmann H, et al. Mean platelet volume is an independent risk factor for myocardial infarction but not for coronary artery disease. Br J Haematol 2002; 117: 399-404.

16. Bath PMW, Butterworth RJ. Platelet size: measurement, physiology and vascular disease. Blood Coagul Fibrinolysis 1996; 7: 157-61.

17. Bath PM, Missouris CG, Buckenham T, et al. Increased platelet volume and platelet mass in patients with atherosclerotic renal artery stenosis. Clin Sci (Lond) 1994; 87: 253-7.

18. Icli A, Aksoy F, Dogan A, et al. Increased mean platelet volume in hypertrophic cardiomyopathy. Angiology 2014; 65: 420-4.

19. Makay B, Turkyilmaz Z, Unsal E. Mean platelet volume in children with familial Mediterranean fever. Clin Rheumatol 2009; 28: 975-8.

20. Karadag-Oncel E, Ozsurekci Y, Kara A, et al. The value of mean platelet volume in the determination of community acquired pneumonia in children. Ital J Pediatr 2013; 39: 16.

21. Ozturk ZA, Dag MS, Kuyumcu ME, et al. Could platelet indices be new biomarkers for inflammatory bowel diseases? Eur Rev Med Pharmacol Sci 2013; 17: 334-41.

22. Liu R, Gao F, Huo J, et al. Study on the relationship between mean platelet volume and platelet distribution width with coronary artery lesion in children with Kawasaki disease. Platelets 2012; 23: 11-6.

23. Kario K, Matsuo T, Nakao K. Cigarette smoking increases the mean platelet volume in elderly patients with risk factors for atherosclerosis. Clin Lab Haematol 1992; 14: 281-7.

24. Khawaja IS, Westermeyer JJ, Gajwani P, Feinstein RE. Depression and coronary artery disease: the association, mechanisms, and therapeutic implications. Psychiatry (Edgmont) 2009; 6: 38-51.

25. Aktas G, Alcelik A, Tekce BK, et al. Red cell distribution width and mean platelet volume in patients with irritable bowel syndrome. Gastroenterol Rev 2014; 9: 160-3.

26. Topal F, Karaman K, Akbulut S, et al. The relationship between mean platelet volume levels and the inflammation in Helicobacter pylori gastritis. J Nat Med Assoc 2010; 102: 726-30.

27. Yeniova A, Kucukazman M, Ata N, et al. Investigation of the association between mean platelet volume and Helicobacter pylori gastritis. Afr J Microbiol Res 2013; 7: 2179-83.

28. Umit H, Umit EG. Helicobacter pylori and mean platelet volume: a relation way before immune thrombocytopenia? Eur Rev Medical and Pharmacological Sciences 2015; 19: 2818-23.

29. Ugras M, Vurmaz A, Tokyol C, et al. Evaluation of the possible relation between Helicobacter pylori gastritis and mean platelet volume: an observational study. Yeditepe Med J 2016; 12: 968-73.

Received: 11.01.2019

Accepted: 7.02 .2019 